\title{
Crystal Structure of a Cytochrome P450 2B6 Genetic Variant in Complex with the Inhibitor 4-(4-Chlorophenyl)imidazole at 2.0-Å Resolution
}

\author{
Sean C. Gay, Manish B. Shah, Jyothi C. Talakad, Keiko Maekawa, Arthur G. Roberts, \\ P. Ross Wilderman, Ling Sun, Jane Y. Yang, Stephanie C. Huelga, Wen-Xu Hong, \\ Qinghai Zhang, C. David Stout, and James R. Halpert
}

Skaggs School of Pharmacy and Pharmaceutical Sciences (S.C.G., M.B.S., J.C.T., A.G.R., P.R.W., J.R.H.), Department of Chemistry and Biochemistry (J.Y.Y.), and Graduate Program in Bioinformatics (S.C.H.), University of California, San Diego, La Jolla, California; Division of Structural Genomics, National Institute of Health Sciences, Tokyo, Japan (K.M.), Department of Surgery, Medical University of South Carolina, Charleston, South Carolina (L.S.); and the Department of Molecular Biology, The Scripps Research Institute, La Jolla, California (W.-X.H., Q.Z., C.D.S.)

Received November 18, 2009; accepted January 8, 2010

\begin{abstract}
The structure of the K262R genetic variant of human cytochrome P450 2B6 in complex with the inhibitor 4-(4-chlorophenyl)imidazole (4-CPI) has been determined using X-ray crystallography to $2.0-\AA$ A resolution. Production of diffraction quality crystals was enabled through a combination of protein engineering, chaperone coexpression, modifications to the purification protocol, and the use of unique facial amphiphiles during crystallization. The 2B6-4-CPI complex is virtually identical to the rabbit 2B4 structure bound to the same inhibitor with respect to the arrangement of secondary structural elements and the placement of active site residues. The structure supports prior P450 2B6 homology models based on other mammalian cytochromes P450 and is consistent with the limited site-directed mutagenesis studies on 2B6 and
\end{abstract}

extensive studies on P450 2B4 and 2B1. Although the K262R genetic variant shows unaltered binding of 4-CPI, altered binding affinity, kinetics, and/or product profiles have been previously shown with several other ligands. On the basis of new P450 2B6 crystal structure and previous 2B4 structures, substitutions at residue 262 affect a hydrogen-bonding network connecting the $G$ and $\mathrm{H}$ helices, where subtle differences could be transduced to the active site. Docking experiments indicate that the closed protein conformation allows smaller ligands such as ticlopidine to bind to the 2B6 active site in the expected orientation. However, it is unknown whether 2B6 undergoes structural reorganization to accommodate bulkier molecules, as previously inferred from multiple P450 2B4 crystal structures.
Cytochromes P450 (P450s) belong to a superfamily of heme-containing monooxygenases and are the predominant enzyme responsible for phase I metabolism of clinically relevant drugs (Wang and Tompkins, 2008). Through the incor-

This work was supported by the National Institutes of Health National Institute of Environmental Health Sciences [Grants ES003619, ES006676]; National Institutes of Health National Institute of General Medical Sciences [Grant GM073197]; National Institutes of Health National Institute of Diabetes and Digestive and Kidney Diseases [Training Grant in Heme and Blood Proteins T32-DK07233]; and the National Institutes of Health National Institute of Biomedical Imaging and Bioengineering [Ruth L. Kirschstein National Research Service Award T32-EB009380].

Article, publication date, and citation information can be found at http://molpharm.aspetjournals.org.

doi:10.1124/mol.109.062570. poration of a single oxygen atom, P450s generate products that are more water-soluble and are either readily excreted in the urine or more amenable substrates for phase II conjugation. Previous studies have demonstrated that many of these enzymes are highly flexible (Domanski and Halpert, 2001; Zhao and Halpert, 2006), allowing them to accommodate a wide range of substrates, including numerous steroids, pharmaceuticals, and environmental pollutants (Johnson and Stout, 2005).

P450 2B enzymes were among the first mammalian P450s to be purified and cloned and have served as a prototype for biochemical and biophysical experiments, as well as studies of substrate specificity and of interactions with the redox

ABBREVIATIONS: P450, cytochrome P450; P450 2B6dH, an N-terminally truncated and modified and C-terminally His-tagged form of cytochrome P450 2B6; P450 2B6dH(Y226H, K262R), an N-terminally truncated and modified and C-terminally His-tagged form of the cytochrome P450 2B6 genetic variant K262R with an internal mutation at position 226; SNP, single nucleotide polymorphism; 4-CPI, 4-(4-chlorophenyl)imidazole; Ni-NTA, nickel-nitrilotriacetic acid; DTT, dithiothreitol; BME, 2-mercaptoethanol; PMSF, phenylmethylsulfonyl fluoride; PDB, Protein Data Bank; RMSD, root-mean-square deviation; 232-chol, $3 \alpha, 7 \alpha, 12 \alpha$-tris[( $\beta$-D-maltopyranosyl)ethyloxy]cholane; RP 73401, cyclopentyloxy- $N$-(3,5dichloro-4-pyridyl)-4-methoxybenzamide. 
partners NADPH-cytochrome $\mathrm{P} 450$ reductase and cytochrome $b_{5}$ (Zhao and Halpert, 2006). There are 28 members of the subfamily, the best characterized being rat $2 \mathrm{~B} 1$ and rabbit $2 \mathrm{~B} 4$. The species, strain, and individual differences in P450 2B function with such substrates as steroids, polychlorinated biphenyls, and chloramphenicol and analogs have made these enzymes an excellent model system for structurefunction analysis (Domanski and Halpert, 2001). However, little attention has been paid to the human 2B6 enzyme in the past because of a lack of selective substrates, inhibitors, and monoclonal antibodies for functional characterization (Ekins et al., 2008; Wang and Tompkins, 2008).

Recent improvements in 2B6 quantification have led to increased interest in the enzyme, and a growing list of clinically relevant substrates. Despite its relatively low expression levels in the liver, 2B6 takes part in the metabolism of approximately 3 to $12 \%$ of all drugs on the market (Wang and Tompkins, 2008), including propofol, efavirenz, diazepam, bupropion, and cyclophosphamide (Zanger et al., 2007). The enzyme is inhibited by ticlopidine, clopidogrel, clotrimazole, itraconazole, sertraline, and raloxifene (Walsky et al., 2006). However, predicting how pharmaceuticals interact with 2B6 has been complicated because of its polymorphic nature. With 28 known alleles leading to different gene products, expression levels and drug metabolism vary widely across populations (Zanger et al., 2007). The most commonly occurring variants are single nucleotide polymorphisms (SNPs) that lead to the Q172H, K262R, and R487C mutants (Zanger et al., 2007). Specifically, the K262R variant shows a greater than 3 -fold increase in the $K_{\mathrm{i}}$ values for clopidogrel, itraconazole, and raloxifene and a 6 -fold increase in the $K_{\mathrm{i}}$ of sertraline (Talakad et al., 2009). Unlike wild-type 2B6, the $\mathrm{K} 262 \mathrm{R}$ variant is not inactivated by phencyclidine (Shebley and Hollenberg, 2007), 17-ethynylestradiol, or efavirenz (Bumpus and Hollenberg, 2008). To predict population differences in steroid, drug, and xenobiotic metabolism, a better understanding of $2 \mathrm{~B} 6$ structure-function relationships is required.

Until now, structural data related to $2 \mathrm{~B} 6$ function have been limited to site-directed mutagenesis studies (Domanski et al., 1999; Spatzenegger et al., 2003), homology models based on other mammalian P450s (Domanski et al., 1999; Wang and Halpert, 2002), or mapping 2B6 residues onto structures of 2B4 (Zhao and Halpert, 2006; Kumar et al., 2007; Talakad et al., 2009), with which 2B6 shares $78 \%$ amino acid sequence identity. In addition, methods that infer enzyme structural data based on molecular properties of known substrates have also proven useful (Ekins et al., 2008). However, X-ray crystallography remains the best tool for obtaining atomic-level details. Numerous mammalian P450 structures have been determined by crystallographic methods (Johnson and Stout, 2005; Zhao and Halpert, 2006; Ekins et al., 2008). However, $2 \mathrm{~B} 6$ and $3 \mathrm{~A} 5$ remained as the only human drug metabolizing $\mathrm{P} 450$ s without crystal structures. There are currently five crystal structures of the highly related 2B4 enzyme (Scott et al., 2003, 2004; Zhao et al., 2006, 2007; Gay et al., 2009). These structures represent four markedly different conformations and indicate that other mammalian P450s might exhibit similar plasticity. For example, a crystal structure of $2 \mathrm{~B} 4$ bound to the inhibitor 4-(4-chlorophenyl)imidazole (4-CPI) (Scott et al., 2004) shows the enzyme in a closed conformation in contrast to open complexes with the larger inhibitors 1-(4-phenyl)benzylimidazole (Gay et al., 2009) or bifonazole (Zhao et al., 2006).

X-ray crystallography requires large amounts of highly pure protein. In the past, obtaining sufficient amounts of protein for growing $2 \mathrm{~B} 6$ crystals has been difficult because of problems with heterologous expression and purification (Hanna et al., 2000). N-terminal truncation and modification (Scott et al., 2001), chaperone coexpression (Mitsuda and Iwasaki, 2006), and internal modifications based on comparison with other P450 2B enzymes (Kumar et al., 2007) have greatly improved the yield of high-quality, purified 2B6. Mutational studies have also led to constructs with greater thermal and chemical stability as well as increased solubility (Kumar et al., 2007). Years of combined research have finally led to a system that allows for the expression, purification, and crystallization of $2 \mathrm{~B} 6$. Here, we present the first crystal structure of $2 \mathrm{~B} 6$ in complex with 4 -CPI at 2.0 - $\AA$ resolution. Along with its 2B4 counterpart, P450 2B6 also provides the first example of two crystal structures of mammalian P450 orthologs bound to the same compound. This unique result allows for very a specific comparison of the rabbit and human enzymes and analysis of subtle differences in their structures.

\section{Materials and Methods}

Materials. Imidazole and pyridine compounds were obtained from Sigma-Aldrich (St. Louis, MO). 5-Cyclo-hexylpentyl- $\beta$-D-maltoside (Cymal-5) was from Anatrace (Maumee, OH). Fifty-kilodalton molecular-weight-cutoff Amicon Ultra filtration devices were from Millipore (Billerica, MA). Clear Strategy Screen I HT-96 was from Molecular Dimensions (Apopka, FL). The pGro7 plasmid was acquired from Takara Bio (Shiba, Japan). Nickel-nitrilotriacetic acid (Ni-NTA), affinity resin was from QIAGEN (Valencia, CA). Macroprep CM cation exchange resin was from Bio-Rad Laboratories (Hercules, CA). Escherichia coli JM109 competent cells were from Stratagene (La Jolla, CA). The synthesis of the facial amphiphile $3 \alpha, 7 \alpha, 12 \alpha$-tris [( $\beta$-D-maltopyranosyl)ethyloxy]cholane (232-chol) was performed essentially according to a method described previously (Zhang et al., 2007) and will be reported in more detail elsewhere.

Mutagenesis, Protein Expression, and Purification. To create the Y226H, K262R double mutant construct, P450 2B6dH was used as a template. The Y226H primers 5 '-CTCTGGCTTCTTGAAACACTTTCCTGGGGCACAC- $3^{\prime}$ and 5'-GTGTGCCCCAGGAAAGTGTTTCAAGAAGCCAGAG- ${ }^{\prime}$ and K262R primers 5' - CCCAGCGCCCCCAGGGACCTCATCGAC-3' and 5'-GTCGATGAGGTC CCTGGGGGCGCTGGG-3' were used in subsequent PCR procedures. DNA sequences were analyzed by the Protein Chemistry Laboratory, University of Texas Medical Branch (Galveston, TX) to confirm the inclusion of both mutations.

Cytochrome P450 2B6dH (Y226H, K262R) was coexpressed with the chaperone GroES/EL (pGro7 plasmid) in E. coli JM109 cells as described previously (Talakad et al., 2009) and purified by modifying the same protocol. The pellet was resuspended in $10 \%$ of the original culture volume in buffer containing $20 \mathrm{mM}$ potassium phosphate, $\mathrm{pH}$ 7.4 at $4^{\circ} \mathrm{C}, 20 \%(\mathrm{v} / \mathrm{v})$ glycerol, $10 \mathrm{mM} \mathrm{BME}$, and $0.5 \mathrm{mM}$ PMSF. The resuspended cells were treated further with lysozyme $(0.2 \mathrm{mg} / \mathrm{ml})$ and stirred for $30 \mathrm{~min}$ at $4^{\circ} \mathrm{C}$, followed by a brief centrifugation for 15 min at $7000 \mathrm{rpm}$ in a JA-14 rotor in an Avanti J-26 XPI Centrifuge (Beckman Coulter, Inc., Fullerton, CA). Spheroplasts were again resuspended in $5 \%$ of the original culture volume in buffer containing $500 \mathrm{mM}$ potassium phosphate, $\mathrm{pH} 7.4$ at $4^{\circ} \mathrm{C}, 20 \%(\mathrm{v} / \mathrm{v})$ glycerol, 10 $\mathrm{mM}$ BME, and $0.5 \mathrm{mM}$ PMSF and were sonicated three times for $45 \mathrm{~s}$ on ice. The membrane pellet was separated by centrifugation for 10 $\mathrm{min}$ at $7000 \mathrm{rpm}$ in the same centrifuge as above and Cymal-5 was 
added to the supernatant at a final concentration of $4.8 \mathrm{mM}$. This was allowed to stir for $30 \mathrm{~min}$ at $4^{\circ} \mathrm{C}$ before ultracentrifugation for 45 min at 41,000 rpm using a fixed-angle Ti 50.2 rotor in an Optima L-80 XP Ultracentrifuge (Beckman Coulter, Inc.). The P450 concentration in the supernatant was determined from the reduced $\mathrm{CO}$ difference spectra.

His-tagged 2B6dH (Y226H, K262R) was purified using Ni-NTA resin in the presence of detergent Cymal-5. The column was washed with buffer containing $100 \mathrm{mM}$ potassium phosphate, $\mathrm{pH} 7.4$ at $4^{\circ} \mathrm{C}$, $100 \mathrm{mM} \mathrm{NaCl}, 20 \%$ (v/v) glycerol, $10 \mathrm{mM}$ BME, $0.5 \mathrm{mM}$ PMSF, 4.8 $\mathrm{mM}$ Cymal-5, and $1 \mathrm{mM}$ histidine, and the protein was eluted using the above buffer containing $60 \mathrm{mM}$ histidine. The P450-containing fractions were pooled and diluted 10 -fold in buffer with $5 \mathrm{mM}$ potassium phosphate, $\mathrm{pH} 7.4$ at $4^{\circ} \mathrm{C}, 20 \%$ (v/v) glycerol, $1 \mathrm{mM}$ EDTA, 0.2 $\mathrm{mM}$ DTT, $0.5 \mathrm{mM}$ PMSF, and $4.8 \mathrm{mM}$ Cymal-5 before loading onto a Macroprep CM cation exchange column. The column was washed using $5 \mathrm{mM}$ potassium phosphate, $\mathrm{pH} 7.4$ at $4^{\circ} \mathrm{C}, 20 \mathrm{mM} \mathrm{NaCl}, 20 \%$ $(\mathrm{v} / \mathrm{v})$ glycerol, $1 \mathrm{mM}$ EDTA, and $0.2 \mathrm{mM}$ DTT and the protein was eluted with high-salt buffer containing $50 \mathrm{mM}$ potassium phosphate, $\mathrm{pH} 7.4$ at $4^{\circ} \mathrm{C}, 500 \mathrm{mM} \mathrm{NaCl}, 20 \%$ (v/v) glycerol, $1 \mathrm{mM}$ EDTA, and 0.2 mM DTT. Protein fractions with the highest $A_{417} / A_{280}$ ratios were pooled, and the $\mathrm{P} 450$ concentration was measured using the reduced CO difference spectrum.

Confirmation of the Double Mutant by Mass Spectrometry. Tryptic peptides were analyzed by capillary liquid chromatographyelectrospray ionization-tandem mass spectrometry using an Agilent 1200 quaternary pump high-performance liquid chromatograph (Agilent Technologies, Inc., Santa Clara, CA) coupled with a linear ion trap mass spectrometer (LTQ XL; Thermo Fisher Scientific Inc., Waltham, MA). Proteolytic digests were injected into solvent A (water/acetonitrile/formic acid, 95:5:0.1), trapped on a $\mathrm{C}_{18}$-packed $100 \times$ $5 \mathrm{~mm}$ fused silica capillary column, and eluted by a segmented gradient program (5-95\% solvent B; water/acetonitrile/formic acid, 5:95:0.1; $50 \mathrm{~min}$ ). An initial broadband MS1 scan was acquired from 400 to $2000 \mathrm{~m} / z$ followed by five MS2 scans of the first through fifth most abundant ions, with dynamic exclusion enabled. Tune Plus 1.0 (Thermo Fisher Scientific Inc.) was used to tune the mass spectrometer to the angiotensin $2+$ ion $(523.7749 \mathrm{~m} / \mathrm{z})$ before running samples. A bovine serum albumin digest was run before running samples to evaluate column performance.

The data were analyzed using InSpect (http://proteomics.ucsd.edu/ Software/Inspect.html), against the FASTA databases for the $2 \mathrm{~B} 6 \mathrm{dH}$ mutants. The resulting data set was summarized using an Inspect $p$-value cutoff of 0.05 and analyzed for quality. The spectra for the peptides containing positions 226 and 262 were manually annotated or verified.

Mass spectrometry analysis yielded 32 peptides across 43 spectra. The spectra and corresponding mass list from ProteinProspector (http://prospector.ucsf.edu) for these peptide hits were used to generate theoretical ions from the proposed peptide sequence, and the mass list was compared with the theoretical ion list. One of the peptides, a 7-mer, contained residue 226. By mass spectrometry analysis and manual annotation of the spectrum, position 226 is histidine and not tyrosine. Another peptide hit, corresponding to a 9-mer, contained the other amino acid of interest at position 262. This residue was verified as arginine, not lysine.

Spectral Titrations of Inhibitor Binding to Mutant and Wild-Type P450 2B6dH and P450 2B4dH. For 4-CPI, the UVvisible spectra were measured with a S2000 single channel chargecoupled device rapid scanning spectrometer (Ocean Optics, Inc., Dunedin, FL.) with L7893 deuterium and halogen light source with fiber optic cable (Hamamatsu, Inc., Bridgewater, NJ). The titration experiments were done in a 1-cm cuvette with magnetic stirring compartment (Hellma USA, Plainview, NY) and a HP40107 U Micro stirring unit (Variomag, Daytona Beach, FL). The 4-CPI binding

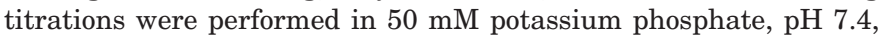
containing $500 \mathrm{mM} \mathrm{NaCl}, 1 \mathrm{mM}$ EDTA, and $0.2 \mathrm{mM}$ DTT with either $20 \%(\mathrm{v} / \mathrm{v})$ glycerol or $500 \mathrm{mM}$ sucrose. Methanol was used as a cosolvent to dissolve 4-CPI and was maintained at a concentration less than $2 \%$ for the titration experiments. When the apparent binding constants $\left(K_{\mathrm{D}}\right)$ were less than the concentration of protein, titration curves were fit to a derivative of the velocity quadratic equation or "tight binding" equation: $A=\left(A_{\max } / 2\left[\mathrm{E}_{\mathrm{T}}\right]\right)\left(\left(\left[\mathrm{E}_{\mathrm{T}}\right]+\left[\mathrm{I}_{\mathrm{T}}\right]+K_{\mathrm{D}}\right)-\right.$ $\left.\left(\left(\left[\mathrm{E}_{\mathrm{T}}\right]+\left[\mathrm{I}_{\mathrm{T}}\right]+K_{\mathrm{D}}\right)^{2}-4\left[\mathrm{E}_{\mathrm{T}}\right]\left[\mathrm{I}_{\mathrm{T}}\right]\right)^{1 / 2}\right)$, where $\left[\mathrm{E}_{\mathrm{T}}\right]$ and $\left[\mathrm{I}_{\mathrm{T}}\right]$ are total enzyme and total inhibitor concentrations, respectively.

Dynamic Light Scattering. The aggregation-dependent light scattering was measured with a DynaPro 96-well plate reader and analyzed with the software package Dynamics version 6.9.2.9 (Wyatt Technology Corporation, Santa Barbara, CA). Each of the samples used for these experiments contained approximately $18 \mu \mathrm{M}$ cytochrome P450 and was in CM-elution buffers containing either 500 $\mathrm{mM}$ sucrose or $20 \%(\mathrm{v} / \mathrm{v})$ glycerol. The soluble bacterial cytochrome $\mathrm{P} 450_{\text {eryF }}$ was used as a monomeric control.

Temperature Stability Assay. Thermal stability of P450 was measured in a reaction mixture containing $1 \mu \mathrm{M}$ protein in $100 \mathrm{mM}$ $\mathrm{NaOH}-\mathrm{HEPES}$ buffer, $\mathrm{pH}$ 7.4. Thermal inactivation was determined from a series of absorbance spectra in the 340- to $700-\mathrm{nm}$ range as a function of temperature between 25 and $70^{\circ} \mathrm{C}$ with intervals of 2.5 to $5^{\circ} \mathrm{C}$ and a 2-min equilibration at each temperature. Determination of total concentration of the heme protein was done by nonlinear leastsquares approximation of the spectra using a linear combination of spectral standards of P450 2B4 low-spin, high-spin, and P420 states. All data treatment and fitting of the titration curves were performed with our SpectraLab software package. Fitting of the temperature profile curves was performed by regression analysis using Sigma Plot. The mid point of thermal transition temperature $\left(T_{\mathrm{m}}\right)$ was obtained by fitting the inactivation profile to sigmoidal two-state model and four-parameter logistic curve (Kumar et al., 2007).

Crystallization and Data Collection. Pooled protein was diluted to $18 \mu \mathrm{M}$ in $50 \mathrm{mM}$ potassium phosphate, $\mathrm{pH} 7.4,500 \mathrm{mM}$ sucrose, $500 \mathrm{mM} \mathrm{NaCl}, 1 \mathrm{mM}$ EDTA, and $0.2 \mathrm{mM}$ DTT. The 4-CPI ligand was added to a final concentration of $180 \mu \mathrm{M}$, and the solution was incubated at $4^{\circ} \mathrm{C}$ overnight. An additional $30 \mathrm{ml}$ of the above buffer containing $180 \mu \mathrm{M}$ 4-CPI was prepared to further dilute the glycerol present with sucrose in the pooled protein fractions. Buffer exchange was performed by concentrating the diluted protein solution to $275 \mu \mathrm{M}$ followed by dilution to $18 \mu \mathrm{M}$, and the process was repeated twice before finally concentrating the 2B6-4-CPI complex to $275 \mu \mathrm{M}$. The concentrated protein-inhibitor complex was supplemented with $4.8 \mathrm{mM}$ Cymal-5, $1 \mathrm{mM}$ 4-CPI, and $0.028 \%$ (w/v) 232 chol (Zhang et al., 2007). Screening was performed by sitting drop vapor diffusion using the Clear Strategy Screen I HT-96 highthroughput kit by mixing equal volumes of the 2B6-4-CPI mixture and well solution. Drops were equilibrated against the well solutions at $18^{\circ} \mathrm{C}$. Crystals for X-ray diffraction analysis were grown from well solution containing $100 \mathrm{mM}$ sodium acetate, $\mathrm{pH} 5.5,200 \mathrm{mM}$ potassium thiocyanate, $10 \%(\mathrm{w} / \mathrm{v})$ polyethylene glycol 1000, and $10 \%(\mathrm{w} / \mathrm{v})$ polyethylene glycol 8000 . Crystals that were approximately $50 \times$ $50 \times 100 \mu \mathrm{m}$ grew over the course of 5 to 7 days. The crystals belonged to the monoclinic space group C121 with a Matthews coefficient of $2.25 \AA^{3} / \mathrm{Da}$ and $45.4 \%$ solvent, assuming one molecule per asymmetric unit.

Crystals were transferred to a solution containing $100 \mathrm{mM}$ sodium acetate, $\mathrm{pH} 5.5,200 \mathrm{mM}$ potassium thiocyanate, $10 \%(\mathrm{w} / \mathrm{v})$ polyethylene glycol 1000, 10\% (w/v) polyethylene glycol 8000, and $500 \mathrm{mM}$ sucrose for cryoprotection before rapid cooling in liquid nitrogen. Initial diffraction patterns at the Stanford Synchrotron Radiation Lightsource (Stanford, CA) showed numerous ice rings. To remove the ice rings, the crystal was annealed by blocking the stream of liquid nitrogen for $4 \mathrm{~s}$ while the crystal was mounted on the goniometer. Two data sets were collected remotely at beamline 11-1 of the Stanford Synchrotron Radiation Lightsource. The first data set was collected using 20-s exposures, and the second data set was collected using 30 -s exposures. Both data sets were collected using $1^{\circ}$ oscillations over $180^{\circ}$ total at $100 \mathrm{~K}$ on a Quantum CCD detector (Area Detector Systems Corp., Poway, CA). Images were integrated 
using iMosflm (Leslie, 1999). The first data set was integrated to 2.0 $\AA$. The second data set was split into two parts; the first 90 frames were integrated to $2.0 \AA$ and the last 90 frames were integrated to 2.1 $\AA$ as the crystal began to decay in the X-ray beam. The three integrated portions were merged in CCP4i and scaled using SCALA (Collaborative Computational Project Number 4, 1994).

Structure Determination and Refinement. A molecular replacement solution was found using an ensemble of all five previously determined 2B4 structures (PDB entries 1suo, 1po5, 2bdm, $2 \mathrm{q} 6 \mathrm{n}$, and $3 \mathrm{~g} 5 \mathrm{n}$ ) with residues 101 to 120 and 205 to 232 omitted as a search model in Phaser (McCoy et al., 2007). These regions correspond to mobile, plastic regions in 2B4 (Zhao et al., 2006; Gay et al., 2009 ) and are expected to behave similarly in 2B6. The initial model was subjected to a rigid body refinement and a restrained refinement using the software REFMAC (Collaborative Computational Project Number 4, 1994) before model building in COOT (Emsley and Cowtan, 2004) began using $2 F_{\mathrm{o}}-F_{\mathrm{c}}$ electron density maps contoured at $1-\sigma$. The heme, 4-CPI, and regions deleted from the molecular replacement model were clearly defined in the electron density. The protein model was modified to reflect amino acid differences between the $2 \mathrm{~B} 4$ and $2 \mathrm{~B} 6$ sequences. The iterative process of model building and refinement was continued until a final $R$ factor of $17.8 \%$ and an $R_{\text {free }}$ of $22.8 \%$ were reached. Refinement statistics are summarized in Table 1. Atomic coordinates and structure factors have been deposited in the Protein Data Bank (accession code 3ibd).

Ligand Docking. Before docking the inhibitor ticlopidine, the 2B6dH (Y226H, K262R) structure file was modified. Any residues not modeled into the experimental electron density were added using COOT and 4-CPI, Cymal-5, and thiocyanate ions were removed from the PDB file. The Autodock4 (Morris et al., 1998) script used to

\section{TABLE 1}

X-ray data collection and refinement statistics

Values for the highest resolution shell are in parentheses.

\begin{tabular}{lc}
\hline Crystal & \\
Space Group & $\mathrm{C} 121$ \\
Unit Cell & $89.57 \AA$ \\
$a$ & $55.74 \AA$ \\
$b$ & $99.09 \AA$ \\
$c$ & $90^{\circ}$ \\
$\alpha=\gamma$ & $96.51^{\circ}$ \\
$\beta$ & \\
Data collection & SSRL BL $11-1$ \\
X-ray source & $0.98 \AA$ \\
Wavelength & $49.32 \AA(2.00)$ \\
Resolution range & 223,616 \\
Total observations & 32,536 \\
Unique observations & $98.3 \%(88.1)$ \\
Completeness & $6.9(4.1)$ \\
Redundancy & $4.6(1.4)$ \\
I/ $\sigma$ & $13.1 \%(48.7)$ \\
$R_{\text {merge }}{ }^{\circ}$ & \\
Refinement statistics & $17.8 \%$ \\
$R$ factor & \\
$R_{\text {free }}$ & $22.8 \%$ \\
RMSD & \\
Bond lengths & $0.018 \AA$ \\
Bond angles & $1.767^{\circ}$ \\
Ramachandran plot & \\
Preferred & $98.1 \%$ \\
Allowed & $1.5 \%$ \\
No. of atoms & \\
Protein & 3791 \\
Heme & 43 \\
4-CPI & 12 \\
Cymal-5 5 Thiocyanate & 22 \\
Water & 12 \\
\hline
\end{tabular}

SSRL BL 11-1, beamline 11-1 of the Stanford Synchrotron Radiation Lightsource. ${ }^{a} R_{\text {merge }}=\left[\Sigma_{\mathrm{h}} \Sigma_{\mathrm{i}} I_{\mathrm{h}}-I_{\mathrm{hi}} / \Sigma_{\mathrm{h}} \Sigma_{\mathrm{i}} I_{\mathrm{hi}}\right]$ where $I_{\mathrm{h}}$ is the mean of $I_{\mathrm{hi}}$ observations of reflection $\mathrm{h}$.

${ }^{b} R$ factor $\& R_{\text {free }}=\Sigma|| F_{\text {obs }}|-| F_{\text {calc }}|| \Sigma\left|F_{\text {obs }}\right| \times 100$ for $95 \%$ of the recorded data $(\mathrm{R}$ factor $)$ and $5 \%$ of data $\left(R_{\text {free }}\right)$. simulate ligand binding added all hydrogen atoms to the protein and ligand PDB files, removed any water molecules, and was run with default settings. Small molecule charges were set to Gasteiger. Charges found on the heme were modified using a separate script citing values previously reported (Helms and Wade, 1995). The docking experiment included 100 events using a grid size of $70 \times 70 \times 70$ $\AA$ with 0.375 -A spacing.

Figures. All protein model figures were generated using PyMOL (DeLano Scientific, Palo Alto, CA).

\section{Results}

Construct Selection. Structural studies of $2 \mathrm{~B} 6$ have lagged behind those on other $2 \mathrm{~B}$ enzymes as a result of low expression levels (Hanna et al., 2000) and low thermal stability compared with 2B1, 2B4, and 2B11 (Kumar et al., 2007). Decreased stability is associated with $\mathrm{P} 420$ formation and aggregation during purification. Initial improvements in expression arose by applying the same $\mathrm{N}$-terminal truncations and modifications used to enhance expression in $2 \mathrm{C} 3$ (von Wachenfeldt et al., 1997) and 2C5 (Cosme and Johnson, 2000) to produce P450 2B6dH (Scott et al., 2001) and by coexpression with the chaperone GroEL/ES (Mitsuda and Iwasaki, 2006). However, the instability of 2B6 caused aggregation during purification and precipitation during crystallization trials.

Efforts to rationally engineer $2 \mathrm{~B} 6 \mathrm{dH}$ mutants based on sequence comparisons with $2 \mathrm{~B} 1,2 \mathrm{~B} 4$, and $2 \mathrm{~B} 11$ have shown that mutants such as $\mathrm{L} 264 \mathrm{~F}$ can be created that exhibit increased expression and thermal stability but retain substrate specificity (Kumar et al., 2007). However, this mutant showed no advantage over $2 \mathrm{~B} 6 \mathrm{dH}$ in crystallization trials. Based on reports of enhanced solubility of the naturally occurring genetic variant K262R (Bumpus et al., 2005), this mutant was also tested. However, the decreased temperature stability was a liability (Table 2). Unfortunately, combining the K262R and L264F mutations resulted in a construct with a lower $T_{\mathrm{m}}$ than the K262R single mutant. A search for other mutants with enhanced stability yielded Y226H, which, like the L264F mutant, maintained sensitivity to inhibition by a variety of ligands (Table 3). The Y226H, K262R double mutant retained the desirable traits from both single mutants, showing increased expression, thermal stability, and solubility. 4-CPI was chosen for crystallization experiments because it is the tightest binding nitrogenous inhibitor of $2 \mathrm{~B} 6$ (Table 3 ) and has yielded the highest resolution ligand complex with 2B4 (Scott et al., 2004). The double mutant also exhibited tight spectral binding of 4-CPI with a $K_{\mathrm{s}}$ value of $0.19 \mu \mathrm{M}$ compared with $0.24 \mu \mathrm{M}$ in $2 \mathrm{~B} 6 \mathrm{dH}$ (data not shown). The combination of the $2 \mathrm{~B} 6 \mathrm{dH}(\mathrm{Y} 226 \mathrm{H}, \mathrm{K} 262 \mathrm{R})$ construct coexpressed with GroEL/ES results in a high yield of quality protein suitable for crystallization experiments.

TABLE 2

Melting temperatures $\left(T_{\mathrm{m}}\right)$ of $2 \mathrm{~B} 6 \mathrm{dH}$ and mutants as determined by thermal inactivation

\begin{tabular}{cc}
\hline Mutant & $T_{\mathrm{m}}$ \\
\hline & ${ }^{\circ} \mathrm{C}$ \\
2B6dH & 48.2 \\
Y226H & 51.4 \\
$\mathrm{~K} 262 \mathrm{R}$ & 46.3 \\
$\mathrm{Y} 226 \mathrm{H} / \mathrm{K} 262 \mathrm{R}$ & 52.0 \\
$\mathrm{~K} 262 \mathrm{R} / \mathrm{L} 264 \mathrm{~F}$ & 44.3 \\
$\mathrm{~L} 264 \mathrm{~F}$ & 52.2 \\
\hline
\end{tabular}


Purification and Crystallization. Initial crystallization trials were performed at $\sim 30 \mathrm{mg}$ protein $/ \mathrm{ml}$, which was suitable for 2B4dH crystallization and that of many other mammalian P450s (Williams et al., 2000; Schoch et al., 2004; Sansen et al., 2007; Porubsky et al., 2008). However, this gave rise to heavy precipitation of the various $\mathrm{P} 4502 \mathrm{~B} 6 \mathrm{dH}$ constructs. Lowering the protein concentration to $15 \mathrm{mg} / \mathrm{ml}$ resulted in a fair balance between clear drops and precipitation and yielded numerous liquid-liquid phase separations, indicating a favorable state for crystallization.

After numerous promising liquid-liquid phase separations, but no crystallization hits, it was clear that altering another parameter was necessary. Based on results with human 2E1dH (Porubsky et al., 2008), the 20\% glycerol in the final 2B6dH(Y226H, K262R) preparation was replaced with $0.5 \mathrm{M}, 1.0 \mathrm{M}$ or $1.5 \mathrm{M}$ sucrose. This resulted in less precipitation during crystallization trials and more liquidliquid phase separation. DLS experiments confirmed that 2B6 remained monomeric in buffers containing sucrose in place of glycerol (data not shown). Most important, the first real crystallization hit was found, consisting of needle clusters from protein samples containing $0.5 \mathrm{M}$ sucrose. Subsequently, 2B6dH(Y226H, K262R) was purified by including the detergent Cymal-5 on the Ni-NTA column and replacing CM-Sepharose resin with Macro-Prep CM Media. This resulted in numerous small needle clusters. Unfortunately, the resulting crystals yielded poor or no diffraction during X-ray experiments. Addition of 232-chol (Zhang et al., 2007) at the critical micelle concentration to the protein solution before mixing with well solutions resulted in new crystal forms under different conditions than those seen without the compound. The resulting crystals would not grow in the absence of this compound. We found a single crystal grown in the presence of 232-chol that diffracted to $2.0 \AA$, whereas others typically diffracted to $\sim 3$ to $4 \AA$.

Structure Determination of the 2B6-4-CPI Complex. The 2B6-4-CPI complex structure was solved using molecular replacement from an ensemble of all five previously solved 2B4 structures. The program Phaser selected the 2B41-CPI structure as the most closely related structure (RMSD $0.8 \AA$ ) with a rotational Z-score of 28.7 , a translational Zscore of 24.6, and no packing clashes. The initial unbiased density clearly showed the heme and 4-CPI bound in the active site. Density for the cyclohexane ring and portions of the aliphatic chain of two Cymal-5 molecules was observed in separate hydrophobic pockets and was modeled just before the final rounds of refinement. The maltose groups were not present in the electron density for either detergent molecule. In addition, four thiocyanate ions were also found in the electron density and were added to the model at the same time. Although 232-chol was necessary for crystallization, it was not observed in the electron density maps. Refinement statistics are summarized in Table 1.

The tertiary structure of $2 \mathrm{~B} 6$ is similar to that of other mammalian P450s, consisting of a large $\alpha$-helical domain and a smaller $\beta$-sheet domain (Fig. 1). The final model is defined in the electron density from Gly 28 (wild-type numbering) through His 492 (the first residue of the $4 \times \mathrm{C}$-terminal His tag). Mutated residue 226 is present on the surface-exposed $\mathrm{G}^{\prime} / \mathrm{G}$ loop and does not form any crystal contacts in the structure. The natural mutant K262R occurs on the G/H loop and forms similar interactions to those found with Arg 262 in 2B4. The formation of four hydrogen bonds among Arg 262, Thr 255, and Asp 266 is likely to aid in stabilizing this loop compared with the wild-type enzyme. This could explain why the genetic variant crystallizes more readily.

Comparison with P450 2B4. The length and placement of secondary structural elements in 2B6 is almost identical to that found in the 2B4-4-CPI complex (Fig. 2). Plastic regions identified in 2B4 (the $\mathrm{B} / \mathrm{C}$ loop and $\mathrm{F}$ through $\mathrm{G}$ helices) adopt similar conformations in 2B6, yielding an RMSD of $0.65 \AA$ in a $\mathrm{C}_{\alpha}$ overlay (Fig. 3a). This small RMSD is even less than that found between the 1-CPI and 4-CPI structures of 2B4 $(0.76 \AA)$, indicating that the ability to conform to these particular ligands outweighs protein sequence differences found between $2 \mathrm{~B} 4$ and $2 \mathrm{~B} 6$. This closed conformation is similar to that also found in other family 2 enzymes: 2C5 (Williams et al., 2000), 2C8 (Schoch et al., 2004), and 2C9 (Williams et al., 2003).

In the 4-CPI complexes of both $2 \mathrm{~B} 6$ and $2 \mathrm{~B} 4$, the heme is held in place through an iron-thiolate bond at residue Cys 436 and is surrounded by the C, I, and L helices. Hydrogen bonds to Arg 98, His 369, and Ser 430 hold the A ring propionate group into place. The $\mathrm{D}$ ring propionate is found to interact with $\operatorname{Arg} 98, \operatorname{Trp} 121, \operatorname{Arg} 125$, and Arg 434. These residues are highly conserved across family 2 enzymes, but alternative conformations in 2B4 have shown variability in the level of heme interaction with these residues (Gay et al., 2009).

The active site of $2 \mathrm{~B} 6$, as defined by residues within a generous $5 \AA$ radius of 4-CPI involves the B/C loop, helices $\mathrm{F}$ and I, and the loops found between helix $\mathrm{K}$ and $\beta$-strand 1-4 and between $\beta$-strands $4-1$ and 4-2 (Figs. 2 and $3 \mathrm{~b}$ ). The

\section{TABLE 3}

$\mathrm{IC}_{50}$ values for various ligands as determined by enzyme inhibition assays

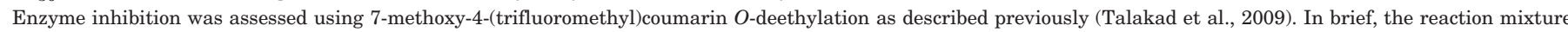

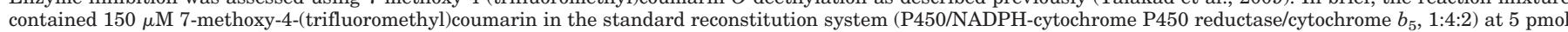

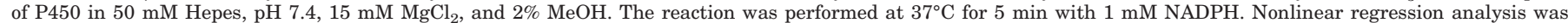
performed to fit the data using a four-parameter logistic function to derive the $\mathrm{IC}_{50}$ values. Standard errors for fitting to the equation are shown.

\begin{tabular}{|c|c|c|c|}
\hline \multirow{2}{*}{ Ligand } & \multicolumn{3}{|c|}{$\mathrm{IC}_{50}$} \\
\hline & $2 \mathrm{~B} 6 \mathrm{dH}$ & $\mathrm{Y} 226 \mathrm{H}$ & $\mathrm{L} 264 \mathrm{~F}$ \\
\hline & \multicolumn{3}{|c|}{$\mu M$} \\
\hline 4-Phenylpyridine & $5.6 \pm 1.6$ & $3.5 \pm 1.3$ & $5.7 \pm 1.5$ \\
\hline 4-(4-Chlorophenyl)pyridine & $0.32 \pm 0.12$ & $0.29 \pm 0.10$ & $0.7 \pm 0.28$ \\
\hline 4-(4-Chlorophenyl)imidazole & $0.26 \pm 0.10$ & $0.27 \pm 0.08$ & $0.37 \pm 0.07$ \\
\hline 1-(4-Chlorophenyl)imidazole & $0.53 \pm 0.013$ & $0.54 \pm 0.14$ & $0.37 \pm 0.05$ \\
\hline 1-Benzylimdazole & $7.5 \pm 1.2$ & $6.2 \pm 0.3$ & $5.5 \pm 1.2$ \\
\hline 4-Phenylimidazole & $1.4 \pm 0.9$ & $2.5 \pm 0.5$ & $1.7 \pm 0.9$ \\
\hline
\end{tabular}


majority of these residues are identical to those that interact with 4-CPI in the 2B4 structure. In 2B6, Val 104, Phe 115, and Glu 301 move slightly out of the 5 - $\AA$ radius used to define the active site (Table 4). Because the orientation of 4-CPI is so similar in both enzymes, the apparent loss of Val 104 as an active site residue in $2 \mathrm{~B} 6$ is tied to a small shift in the angle 4-CPI makes with the plane of the heme. Specifically, the $\sim 2^{\circ}$ increase in this angle in 2B6 leads to Val 104 being an additional $0.45 \AA$ away from the inhibitor. The movement of Phe 115 seems to be linked to small changes in the location and orientation of other residues in the B/C loop. On one side, Ile 101 flips approximately $180^{\circ}$ around X-1 in $2 \mathrm{~B} 6$, pushing a methyl group into space occupied by the phenyl ring of Phe 115 in 2B4. On the other side, Phe 108 shifts toward the G helix to accommodate the movement of Phe 115. The carboxylate group of Glu 301 in 2B4 hydrogen bonds to one of the nitrogens of the ligand imidazole ring. In 2B6, Glu 301 flips
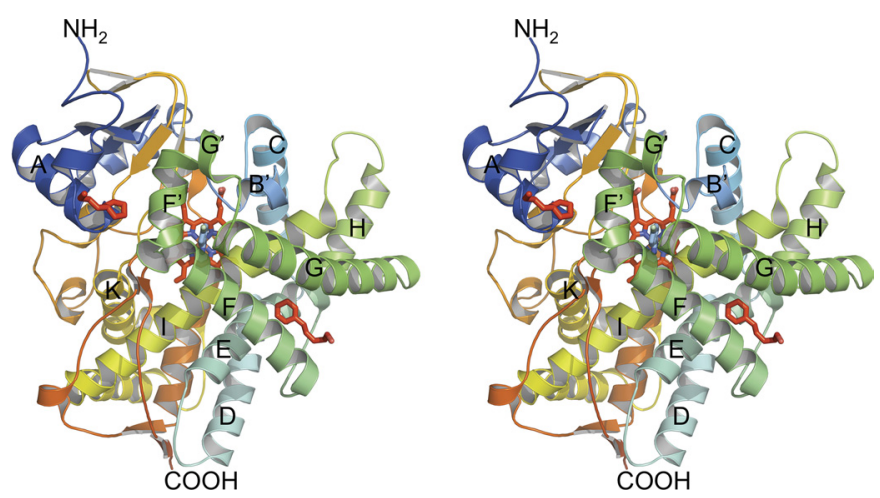

Fig. 1. Divergent stereo view of the 2B6-4-CPI complex. The sequence is colored from the $\mathrm{N}$ terminus (blue) to the $\mathrm{C}$ terminus (red). The heme and partial Cymal-5 detergents are shown as red sticks. The 4-CPI inhibitor is shown as cyan sticks. out of the active site to interact with a water molecule. A similar difference is seen between the 2B4-4-CPI and-1-CPI complexes, indicating that the interaction with Glu 301 is not necessary for CPI binding. However, the additional hydrogen bond could help explain subtle differences in CPI binding in human and rabbit enzymes, because 2B4 binds 4-CPI with a $K_{\mathrm{s}}$ value of $0.04 \mu \mathrm{M}$ (Scott et al., 2004) and $2 \mathrm{~B} 6 \mathrm{dH}(\mathrm{Y} 226 \mathrm{H}$, $\mathrm{K} 262 \mathrm{R}$ ), a value of $0.19 \mu \mathrm{M}$. Phe 206 on the $\mathrm{F}$ helix swings into the active site to fill some of the void left behind by the movement of Glu 301. In addition, residue 363 is an isoleucine in $2 \mathrm{~B} 4$, but a leucine in $2 \mathrm{~B} 6$.

Ligand Docking. To evaluate the potential utility of the new 2B6 model for future ligand docking experiments, we tested the known inhibitor ticlopidine. Ticlopidine oriented itself readily in the active site without any steric clashes. After 100 Autodock runs, ticlopidine docking resulted in five clusters of poses. The two most populated clusters included 81 poses with the thiophene ring oriented toward the heme (Fig. 4) and mean binding energies of -7.67 and $-7.57 \mathrm{kcal} /$ mol, respectively. These poses are consistent with $2 \mathrm{~B} 6$-mediated oxidation of ticlopidine (Nishiya et al., 2009), and each 4-CPI contact residue listed in Table 4 falls within a 5 - $\AA$ radius of the docked inhibitor. In addition, 16 poses showed alternate ligand orientations and mean energies from -7.57 to $-7.27 \mathrm{kcal} / \mathrm{mol}$.

\section{Discussion}

Before the determination of the $2 \mathrm{~B} 6$ structure, efforts to identify active site residues and other residues responsible for substrate recognition and specificity were limited to mutagenesis studies and homology models based on other mammalian P450s. Of the 10 residues within $5 \AA$ of 4 -CPI in the $2 \mathrm{~B} 6$ active site, residues $209,363,367$, and 477 were shown

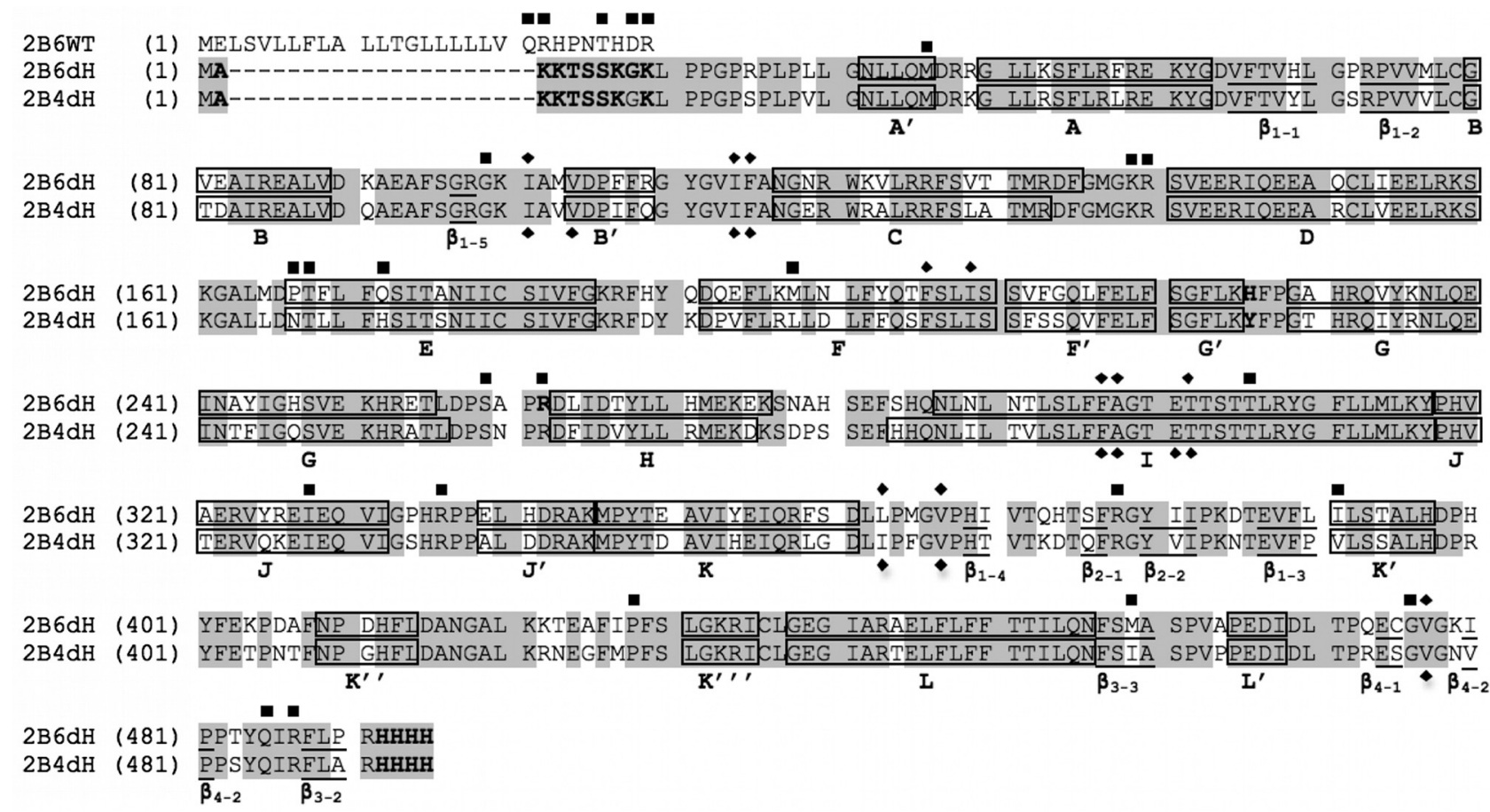

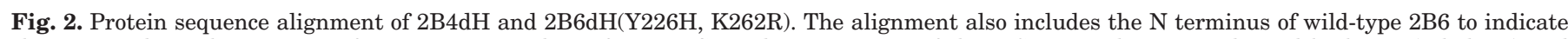

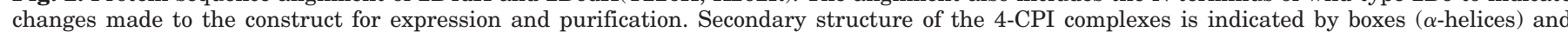

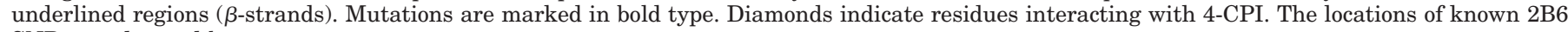
SNPs are denoted by squares. 
to be important for the oxidation of 7-butoxycoumarin (Spatzenegger et al., 2003), and residues 363 and 477 were shown to be important for the oxidation of RP 73401 (Domanski et al., 1999). A homology model based on the structure of 2C5 and data gathered from mutagenesis of 2B1 (Wang and Halpert, 2002) identified residues 114, 115, 206, 209, 297, 298, $302,363,367$, and 477 as taking part in substrate recognition. Residues 103, 290, 294, 478, and 480 are also shown in the active site of this model; these residues, however, do not interact with 4-CPI in the crystal structure. The omission of 4-CPI interaction partners or the inclusion of residues found outside the 4-CPI binding site in these models probably highlights the variability of P450 active sites when confronted with substrates of different sizes and shapes. Overall, mapping residues onto models based on previously determined 2B4 crystal structures (Zhao and Halpert, 2006; Kumar et al., 2007; Talakad et al., 2009) has resulted in the most accurate prediction of the location of $2 \mathrm{~B} 6$ residues.

Among 2B6 active site residues of particular interest is Leu 363 , which is Ile in 2B4 and Val in 2B1. In 2B6, CG1 and CD1 of Leu 363 mimic the corresponding atoms of Ile 363 in 2B4 (Fig. 3b) so that the identity of this residue does not alter the orientation of 4-CPI. However, previous studies have shown that mutations of Leu 363 in 2B6 decrease oxidation of 7-butoxycoumarin, $\mathrm{RP} 73401$, and $p$-nitrophenol and increase 7-ethoxy-4-trifluoromethylcoumarin $O$-deethylase activity (Domanski et al., 1999; Spatzenegger et al., 2003). Other mutational studies at this position have shown a drastic effect on the activation of anticancer drugs by 2B1, 2B4, and 2B5 (Chen et al., 2004) and the metabolism of steroids by 2B1, 2B2, 2B4, 2B5, and 2B11 (Domanski and Halpert, 2001). Leu 363 was also found within a 5 - $\AA$ radius of the ligand in ticlopidine docking simulations. Enzymatic studies of $2 \mathrm{~B}$ mutants combined with additional crystal structures of ligand complexes could help explain the functional role of residue 363 in different species.

Although most of the residues that define the 4-CPI bind- ing site in 2B6 are hydrophobic, there are two residues with polar side chains, Glu 301 and Thr 302. In the 4-CPI complex with 2 B4, Glu 301 makes a hydrogen bond with a free azole nitrogen of the ligand (Scott et al., 2004). However, in the 4-CPI complex with 2B6, Glu 301 moves out of the active site and contacts a nearby water molecule. It is noteworthy that in the 2B4 complex with 1-CPI, which lacks a free nitrogen for hydrogen bonding, Glu 301 also moves out of the active site, in this case interacting with His 172 (Zhao et al., 2007), which is Gln in 2B6. In the 2B6 structure, the apparent small shift in 4-CPI orientation toward Phe 206 (Fig. 3b) is allowed because Glu 301 does not occupy the space that it does in the 2B4 complex. Residue 172 is a known SNP location in 2B6, the $\mathrm{Q} 172 \mathrm{H}$ mutant being most prevalent (Zanger et al., 2007). Because Glu 301 may interact with the side chain of residue 172 , the identity of that residue, along with additional nearby SNP residues 167 and 168 (Fig. 5), could affect ligand binding.

Additional SNPs even farther away from the active site also affect substrate specificity and catalysis. The $2 \mathrm{~B} 6 \mathrm{~K} 262 \mathrm{R}$ genetic variant occurs to varying degrees in human populations. Frequencies typically range from 17 to $63 \%$ (Zanger et al., 2007). With increasing numbers of investigations reporting altered drug metabolism for this and other SNP gene products in $2 \mathrm{~B} 6$, it is imperative to determine how these mutations affect enzyme structure-function relations. The differences found in the pattern of heme pyrrolephenyldiazene adducts indicate that the active site topology of K262R could differ from wild-type (Bumpus and Hollenberg, 2008). Without a wild-type structure for reference, it is impossible to describe those differences in atomic detail at this time. However, most SNPs in 2B6 occur far from the active site (Fig. 5), indicating that any functional effects are transduced via long-range hydrogenbonding networks or through subtle differences in the placement of secondary structural elements.

The K262R mutation occurs on the $\mathrm{G} / \mathrm{H}$ loop, approxi-
A
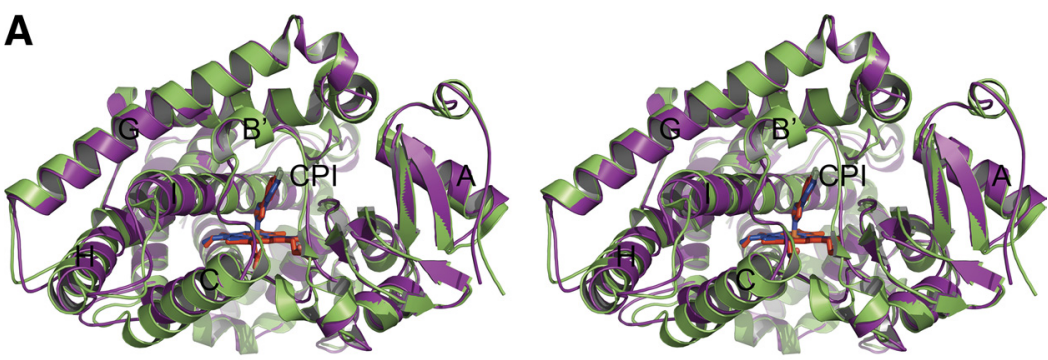

B

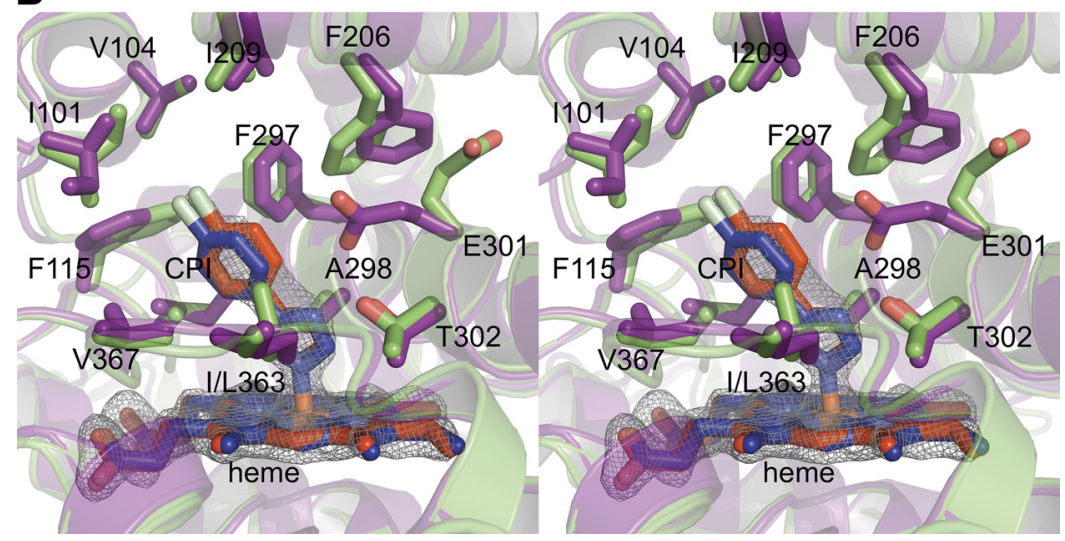

Fig. 3. Divergent stereo view of an overlay of both $2 \mathrm{~B} 4$ (purple) and 2B6 (green) 4-CPI complexes. The 2B6 heme and 4-CPI are shown in red, whereas the corresponding molecules in 2B4 are shown in blue. a, the overall structure of the human and rabbit enzymes, with an RMSD of $0.65 \AA$, are almost indistinguishable from one another. $b$, the active sites of $2 \mathrm{~B} 4$ and $2 \mathrm{~B} 6$ are shown. Residues found within a 5 - $\AA$ radius of 4-CPI are depicted as sticks. An $F_{0}-F_{c}$ simulated annealing omit map contoured at 3- $\sigma$ clearly shows the 2B6 4-CPI and heme. 
TABLE 4

Distances from 4-CPI to the nearest atoms in residues within $5 \AA$ of the inhibitor

\begin{tabular}{|c|c|c|}
\hline Residue & $2 \mathrm{~B} 4$ & $2 \mathrm{~B} 6$ \\
\hline & \multicolumn{2}{|c|}{$\AA$} \\
\hline Ile 101 & 3.59 & 3.95 \\
\hline Val 104 & 4.70 & 5.16 \\
\hline Ile 114 & 4.14 & 4.14 \\
\hline Phe 115 & 3.93 & 5.04 \\
\hline Phe 206 & 6.60 & 4.11 \\
\hline Ile 209 & 5.37 & 4.70 \\
\hline Phe 297 & 3.43 & 3.43 \\
\hline Ala 298 & 3.44 & 3.77 \\
\hline Glu 301 & 3.31 & 6.40 \\
\hline Thr 302 & 3.25 & 3.05 \\
\hline Ile/Leu 363 & 3.71 & 4.00 \\
\hline Val 367 & 3.74 & 3.69 \\
\hline Val 477 & 4.07 & 4.34 \\
\hline
\end{tabular}

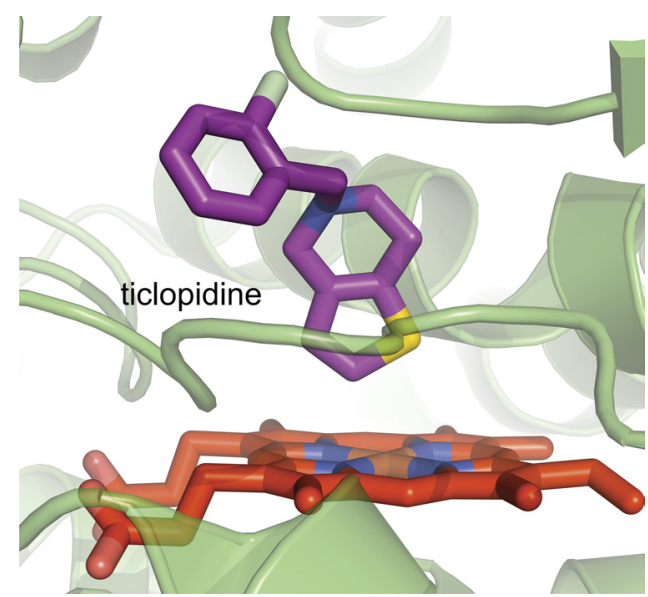

Fig. 4. Ribbon-and-stick depiction of a typical pose for ticlopidine docking into the active site of a model of P450 2B6 based on the crystal structure of the 4-CPI complex. This and other similar poses are consistent with data that show ticlopidine to be oxidized on the thiophene ring and cause mechanism-based inactivation of P450 2B6.

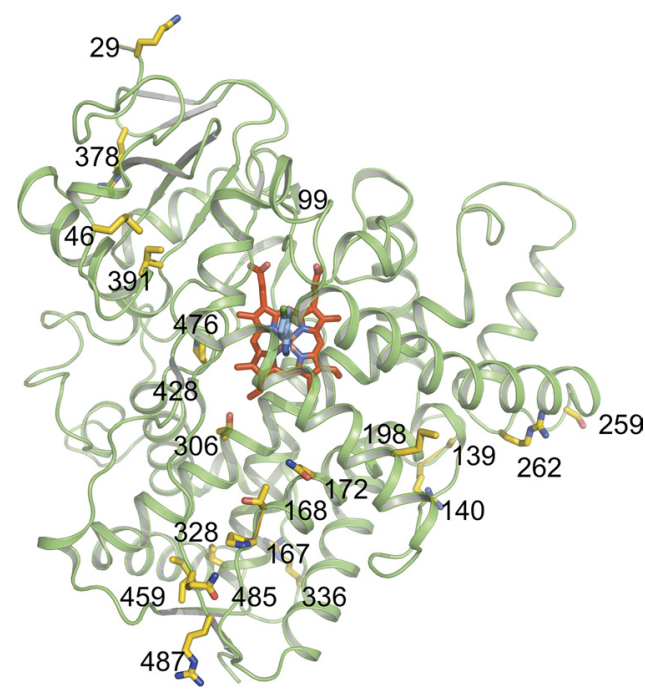

Fig. 5. Ribbon diagram showing the location of known SNPs (yellow sticks) in 2B6. Residues 99 and 476 (both glycine) are not shown as sticks, but locations are identified. The heme is shown as red sticks; 4-CPI, cyan sticks. The majority of the known 2B6 coding sequence variants contain substitutions that occur relatively far from the active site, and none of them actually lies within the active site. mately $25 \AA$ from the active site and does not affect the $\mathrm{IC}_{50}$ (Talakad et al., 2009) or $K_{\mathrm{s}}$ of 4-CPI (data not shown). However, differences have been found with other ligands (Shebley and Hollenberg, 2007; Talakad et al., 2009). A direct connection to the active site through polar interactions could not be found in the current structure. It is noteworthy that the local environment of residue 262 is highly conserved. In both 2B4 and $2 \mathrm{~B} 6 \mathrm{dH}$ (H226Y, K262R), Arg 262 is part of a small hydrogen-bonding network that includes His 252, Thr 255, Asp 263, and Asp 266. An alignment of 10 2B enzymes (not shown) indicates that the only divergence in this cluster of residues is Lys 262 in 2B6. Despite the high degree of mobility of this region in the series of 2B4 crystal structures (Scott et al., 2004; Zhao et al., 2006; Gay et al., 2009), the residues in this network maintain their interactions in every 2B4 structure (Fig. 6) and the present 2B6 structure as well, indicating that the $\mathrm{G}$ and $\mathrm{H}$ helices move in concert. The concerted movement of the $\mathrm{G}$ and $\mathrm{H}$ helices, which could be affected by this hydrogen-bonding network, could influence the orientation of active site residues on the G helix. Alternatively, the identity of residue 262 was previously found to affect $2 \mathrm{~B} 6$ affinity for cytochrome $\mathrm{P} 450$ reductase, but not by an appreciable amount (Bumpus and Hollenberg, 2008). Perhaps interactions between the $\mathrm{H}$ helix and $\mathrm{P} 450$ redox partners are transduced to the G helix and thus to the active site through this connection. Replacing Arg 262 with a lysine, as in wild-type $2 \mathrm{~B} 6$, breaks this network of hydrogen bonds, probably destabilizing this region and could affect either of these hypothesized scenarios.

The 4-CPI complexes of $2 \mathrm{~B} 6$ and $2 \mathrm{~B} 4$ represent the only known case of two crystal structures of mammalian P450 orthologs bound to the same ligand. Comparisons between

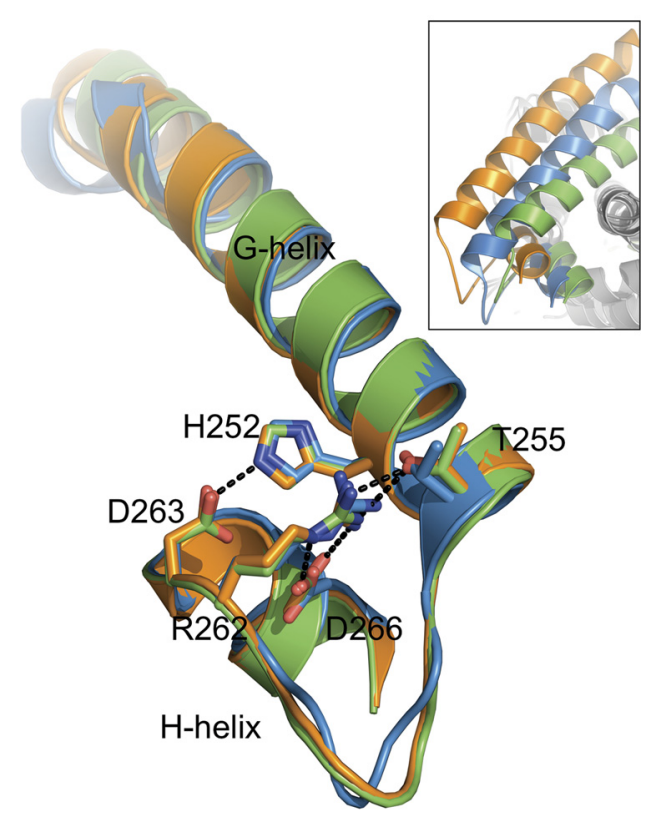

Fig. 6. Ribbon-and-stick diagram depicting the $\mathrm{G}$ and $\mathrm{H}$ helices region in the $2 \mathrm{~B} 4$ bifonazole (orange; $2 \mathrm{bdm}$ ), 1-(4-phenyl)benzylimidazole (1-PBI) (blue; 3g5n), and 4-CPI (green; 1suo) structures. Aligning the $\mathrm{G}$ and $\mathrm{H}$ helices in the absence of the remaining protein shows that this region maintains a consistent local structure despite larger movements in the overall protein (inset). The hydrogen-bonding network among His 252, Thr 255, Arg 262, Asp 263, and Asp 266 seems to link the G and H helices together throughout these shifts in secondary structure placement. These same interactions are also seen in the present $2 \mathrm{~B} 6$ structure. 
the rabbit-human 2B4/2B6 4-CPI pair and 2C5/2C9 unliganded pair (Cosme and Johnson, 2000; Williams et al., 2003) reveal that the $2 \mathrm{~B}$ species differences are much less pronounced than in the $2 \mathrm{C}$ subfamily. The pair of $2 \mathrm{C}$ structures shows much larger variation in the $\mathrm{B} / \mathrm{C}$ loop, $\mathrm{F}$ through $\mathrm{G}$ helices, $\mathrm{H} / \mathrm{I}$ loop, and the $\beta_{4-1}$ and $\beta_{4-2}$ region. However, an exact comparison cannot be made in the case of the $2 \mathrm{C}$ enzymes because the structures are not held into a rigid conformation by a ligand as in the $2 \mathrm{~B}$ case. The differences in the areas mentioned above in the $2 \mathrm{C}$ enzymes could arise from species differences in the protein sequence, but they could also be a result of crystal packing. It is noteworthy that even though the 2B4 and 2B6 crystals were grown under different conditions, the thermodynamics of the 2B-4-CPI complex formation overcomes any differences caused by the $\mathrm{pH}$ and salt content of the crystallization solution as well as crystal lattice contacts. If the $2 \mathrm{C}$ differences are sequencebased and not an effect of crystal packing, structural variations could give insight into enzyme evolution. It is possible that changes in the 2B4/2B6 pair have occurred more slowly than the less structurally related $2 \mathrm{C} 5 / 2 \mathrm{C} 9$ pair.

In conclusion, the $2.0 \AA$ structure of $2 \mathrm{~B} 6 \mathrm{dH}(\mathrm{Y} 226 \mathrm{H}$, $\mathrm{K} 262 \mathrm{R})$ in complex with the inhibitor 4-CPI is the first view of this human enzyme that has been gaining relevance as the list of important xenobiotic substrates has grown. Given the difference of $\sim 100$ amino acids in their sequences, the striking structural similarities between 2B6 and 2B4 were surprising. In this case, the strong interactions with 4-CPI seem to be the driving force to assume the observed conformation and overwhelm any differences that are due to amino acid substitutions. This particular conformation of P450 2B6 is amenable for the docking the small molecule ticlopidine with accurate results and should be a valuable tool for designing new drugs devoid of metabolic liabilities as a result of 2B6mediated oxidation or $2 \mathrm{~B} 6$ inhibition. Additional crystal structures are necessary to determine whether the similarities between $2 \mathrm{~B} 6$ and $2 \mathrm{~B} 4$ persist in the presence of larger ligands that presumably require $2 \mathrm{~B} 6$ to adopt more open conformations. Alternatively, it may be possible to use 2B4 structures as a mimetic for 2B6, given their structural similarities and the superior behavior of 2B4 during purification and crystallization. Further studies, particularly those focused on the role of residue 363 , will be required to test the validity of this proposal.

\section{Acknowledgments}

We thank Dr. Michael Baksh at The Scripps Research Institute for assistance with dynamic light scattering. We also thank the staff at the Stanford Synchrotron Radiation Lightsource for assistance with data collection. The Stanford Synchrotron Radiation Lightsource is operated by Stanford University on behalf of the United States Department of Energy, Office of Basic Energy Sciences. The Stanford Synchrotron Radiation Lightsource is supported by the National Institute of Health, the National Center for Research Resources, the Biomedical Technology Program, and the United States Department of Energy of Biological and Environmental Research.

\section{References}

Bumpus NN and Hollenberg PF (2008) Investigation of the mechanisms underlying the differential effects of the K262R mutation of P450 2B6 on catalytic activity. Mol Pharmacol 74:990-999.

Bumpus NN, Sridar C, Kent UM, and Hollenberg PF (2005) The naturally occurring cytochrome P450 (P450) 2B6 K262R mutant of P450 2B6 exhibits alterations in substrate metabolism and inactivation. Drug Metab Dispos 33:795-802.

Chen CS, Lin JT, Goss KA, He YA, Halpert JR, and Waxman DJ (2004) Activation of the anticancer prodrugs cyclophosphamide and ifosfamide: identification of cytochrome P450 2B enzymes and site-specific mutants with improved enzyme kinetics. Mol Pharmacol 65:1278-1285.

Collaborative Computational Project Number 4 (1994) The CCP4 suite: programs for protein crystallography. Acta Crystallogr D Biol Crystallogr 50:760-763.

Cosme J and Johnson EF (2000) Engineering microsomal cytochrome P450 2C5 to be a soluble, monomeric enzyme: mutations that alter aggregation, phospholipid dependence of catalysis, and membrane binding. J Biol Chem 275:2545-2553.

Domanski TL and Halpert JR (2001) Analysis of mammalian cytochrome P450 structure and function by site-directed mutagenesis. Curr Drug Metab 2:117-137.

Domanski TL, Schultz KM, Roussel F, Stevens JC, and Halpert JR (1999) Structurefunction analysis of human cytochrome P-450 2 B6 using a novel substrate, sitedirected mutagenesis, and molecular modeling. J Pharmacol Exp Ther 290:11411147.

Ekins S, Iyer M, Krasowski MD, and Kharasch ED (2008) Molecular characterization of CYP2B6 substrates. Curr Drug Metab 9:363-373.

Emsley P and Cowtan K (2004) Coot: model-building tools for molecular graphics. Acta Crystallogr D Biol Crystallogr 60:2126-2132.

Gay SC, Sun L, Maekawa K, Halpert JR, and Stout CD (2009) Crystal structures of cytochrome P450 2B4 in complex with the inhibitor 1-biphenyl-4-methyl- $1 H$ imidazole: ligand-induced structural response through $\alpha$-helical repositioning. Biochemistry 48:4762-4771.

Hanna IH, Reed JR, Guengerich FP, and Hollenberg PF (2000) Expression of human cytochrome P450 2B6 in Escherichia coli: characterization of catalytic activity and expression levels in human liver. Arch Biochem Biophys 376:206-216.

Helms V and Wade RC (1995) Thermodynamics of water mediating protein-ligand interactions in cytochrome P450cam: a molecular dynamics study. Biophys $J$ 69:810-824.

Johnson EF and Stout CD (2005) Structural diversity of human xenobiotic metabolizing cytochrome P450 monooxygenases. Biochem Biophys Res Commun 338:331-336.

Kumar S, Zhao Y, Sun L, Negi SS, Halpert JR, and Muralidhara BK (2007) Rational engineering of human cytochrome P450 2B6 for enhanced expression and stability: importance of a Leu264->Phe substitution. Mol Pharmacol 72:1191-1199.

Leslie AG (1999) Integration of macromolecular diffraction data. Acta Crystallogr D Biol Crystallogr 55:1696-1702.

McCoy AJ, Grosse-Kunstleve RW, Adams PD, Winn MD, Storoni LC, and Read RJ (2007) Phaser crystallographic software. J Appl Crystallogr 40:658-674.

Mitsuda M and Iwasaki M (2006) Improvement in the expression of CYP2B6 by co-expression with molecular chaperones GroES/EL in Escherichia coli. Protein Expr Purif 46:401-405.

Morris GM, Goodsell DS, Halliday RS, Huey R, Hart WE, Belew RK, and Olson AJ (1998) Automated docking using a Lamarckian genetic algorithm and empirical binding free energy function. $J$ Comput Chem 19:1639-1662.

Nishiya Y, Hagihara K, Ito T, Tajima M, Miura S, Kurihara A, Farid NA, and Ikeda $\mathrm{T}$ (2009) Mechanism-based inhibition of human cytochrome P450 $2 \mathrm{~B} 6$ by ticlopidine, clopidogrel, and the thiolactone metabolite of prasugrel. Drug Metab Dispos 37:589-593.

Porubsky PR, Meneely KM, and Scott EE (2008) Structures of human cytochrome P450 2E1. Insights into the binding of inhibitors and both small molecular weight and fatty acid substrates. J Biol Chem 283:33698-33707.

Sansen S, Yano JK, Reynald RL, Schoch GA, Griffin KJ, Stout CD, and Johnson EF (2007) Adaptations for the oxidation of polycyclic aromatic hydrocarbons exhibited by the structure of human P450 1A2. J Biol Chem 282:14348-14355.

Schoch GA, Yano JK, Wester MR, Griffin KJ, Stout CD, and Johnson EF (2004) Structure of human microsomal cytochrome P450 2C8. Evidence for a peripheral fatty acid binding site. J Biol Chem 279:9497-9503.

Scott EE, He YA, Wester MR, White MA, Chin CC, Halpert JR, Johnson EF, and Stout CD (2003) An open conformation of mammalian cytochrome P450 2B4 at 1.6-A resolution. Proc Natl Acad Sci U S A 100:13196-13201.

Scott EE, Spatzenegger M, and Halpert JR (2001) A truncation of 2 B subfamily cytochromes P450 yields increased expression levels, increased solubility, and decreased aggregation while retaining function. Arch Biochem Biophys 395:57-68.

Scott EE, White MA, He YA, Johnson EF, Stout CD, and Halpert JR (2004) Structure of mammalian cytochrome P450 2B4 complexed with 4-(4-chlorophenyl)imidazole at 1.9- $\AA$ resolution: insight into the range of $\mathrm{P} 450$ conformations and coordination of redox partner binding. J Biol Chem 279:27294-27301.

Shebley M and Hollenberg PF (2007) Mutation of a single residue (K262R) in P450 2B6 leads to loss of mechanism-based inactivation by phencyclidine. Drug Metab Dispos 35:1365-1371.

Spatzenegger M, Liu H, Wang Q, Debarber A, Koop DR, and Halpert JR (2003) Analysis of differential substrate selectivities of CYP2B6 and CYP2E1 by sitedirected mutagenesis and molecular modeling. J Pharmacol Exp Ther 304:477487.

Talakad JC, Kumar S, and Halpert JR (2009) Decreased susceptibility of the cytochrome P450 2B6 variant K262R to inhibition by several clinically important drugs. Drug Metab Dispos 37:644-650.

von Wachenfeldt C, Richardson TH, Cosme J, and Johnson EF (1997) Microsomal P450 2C3 is expressed as a soluble dimer in Escherichia coli following modification of its N-terminus. Arch Biochem Biophys 339:107-114.

Walsky RL, Astuccio AV, and Obach RS (2006) Evaluation of 227 drugs for in vitro inhibition of cytochrome P450 2B6. J Clin Pharmacol 46:1426-1438.

Wang H and Tompkins LM (2008) CYP2B6: new insights into a historically overlooked cytochrome P450 isozyme. Curr Drug Metab 9:598-610.

Wang Q and Halpert JR (2002) Combined three-dimensional quantitative structureactivity relationship analysis of cytochrome P450 2B6 substrates and protein homology modeling. Drug Metab Dispos 30:86-95.

Williams PA, Cosme J, Sridhar V, Johnson EF, and McRee DE (2000) Microsoma cytochrome P450 2C5: comparison to microbial P450s and unique features. J Inorg Biochem 81:183-190. 
Williams PA, Cosme J, Ward A, Angove HC, Matak Vinković D, and Jhoti H (2003) Crystal structure of human cytochrome P450 2C9 with bound warfarin. Nature 424:464-468.

Zanger UM, Klein K, Saussele T, Blievernicht J, Hofmann MH, and Schwab M (2007) Polymorphic CYP2B6: molecular mechanisms and emerging clinical significance. Pharmacogenomics 8:743-759.

Zhang Q, Ma X, Ward A, Hong WX, Jaakola VP, Stevens RC, Finn MG, and Chang G (2007) Designing facial amphiphiles for the stabilization of integral membrane proteins. Angew Chem Int Ed Engl 46:7023-7025.

Zhao Y and Halpert JR (2006) Structure-function analysis of cytochromes P450 2B. Biochim Biophys Acta 1770:402-412.

Zhao Y, Sun L, Muralidhara BK, Kumar S, White MA, Stout CD, and Halpert JR
(2007) Structural and thermodynamic consequences of 1-(4-chlorophenyl)imidazole binding to cytochrome P450 2B4. Biochemistry 46:11559-11567.

Zhao Y, White MA, Muralidhara BK, Sun L, Halpert JR, and Stout CD (2006) Structure of microsomal cytochrome P450 2B4 complexed with the antifungal drug bifonazole: insight into $\mathrm{P} 450$ conformational plasticity and membrane interaction. J Biol Chem 281:5973-5981.

Address correspondence to: Sean C. Gay, Skaggs School of Pharmacy and Pharmaceutical Sciences, University of California, San Diego, 9500 Gilman Dr. \#0703, La Jolla, CA 92093. E-mail: scgay@ucsd.edu 